\title{
New result of Antideuteron search in BESS-Polar II
}

K.Sakai, ${ }^{a, i, *}$ K. Abe ${ }^{*}, b$ H. Fuke, ${ }^{c}$ S. Haino ${ }^{\dagger},{ }^{d}$ T. Hams, ${ }^{a, i}$ M. Hasegawa, ${ }^{d}$ K. C. Kim, ${ }^{e}$ M. H. Lee, ${ }^{e}$ Y. Makida, ${ }^{d}$ J. W. Mitchell, ${ }^{a}$ J. Nishimura, ${ }^{f}$ M. Nozaki, ${ }^{d}$ R. Orito ${ }^{\ddagger}, b$ J. F. Ormes, ${ }^{g}$ N. Picot-Clemente, ${ }^{e}$ M. Sasaki, ${ }^{a, i}$ E. S. Seo,${ }^{e}$ R. E. Streitmatter ${ }^{\S},{ }^{a}$ N. Thakur, ${ }^{a}$ A. Yamamoto, ${ }^{d}$ T. Yoshida ${ }^{c}$ and K. Yoshimura ${ }^{h}$

${ }^{a}$ NASA-Goddard Space Flight Center (NASA-GSFC), Greenbelt,MD 20771, USA

${ }^{b}$ Kobe University, Kobe, Hyogo 657-8501, Japan

${ }^{c}$ Institute of Space and Astronautical Science, Japan Aerospace Exploration Agency (ISAS/JAXA), Sagamihara, Kanagawa 252-5210, Japan

${ }^{d}$ High Energy Accelerator Research Organization (KEK), Tsukuba, Ibaraki 305-0801, Japan

e IPST, University of Maryland, College Park, MD 20742, USA

${ }^{f}$ The University of Tokyo, Bunkyo, Tokyo 113-0033, Japan

${ }^{g}$ University of Denver, Denver, CO 80208, USA

${ }^{\text {h}}$ Okayama University, Okayama, Okayama 700-0082, Japan

${ }^{i}$ Center for Research and Exploration in Space Science and Technology (CRESST)

E-mail: kenichi.sakai@nasa.gov

High precision cosmic-ray low energy antiproton fluxes reported by BESS-Polar, PAMELA and AMS-02 are consistent with secondary production from interactions of primary cosmic rays with the interstellar medium. This severely constrains the possibility of antiprotons of primary origin such as annihilation or decay of supersymmetric dark matter or evaporation of primordial black holes. In the case of antideuterons, secondary production in collisions is strongly suppressed, especially at low energies, because of the very low production cross-section and strict kinematic requirements compared to antiproton production. The lack of secondary background would imply that there is still plenty of room to search for primary antideuterons from novel production processes. The most sensitive search ever realized was reported by using the BESS flight data obtained during the previous solar minimum period in 1997. By comparison, the BESS-Polar II flight in 2007/2008 accumulated cosmic-ray data in near solar minimum conditions with more than ten times the statistics of BESS97. We will report the result of a new search for antideuterons with unprecedented sensitivity by using BESS-Polar II data.

$37^{\text {th }}$ International Cosmic Ray Conference (ICRC 2021)

July 12 th - 23rd, 2021

Online-Berlin, Germany

\footnotetext{
*Present address: Kamioka Observatory, ICRR, The University of Tokyo, Hida, Gifu 506-1205, Japan.

$\dagger$ Present address: Institute of Physics, Academia Sinica, Nankang, Taipei 11529, Taiwan.

†Present address: Tokushima University, Tokushima, Tokushima 770-8502, Japan.

$\S$ deceased

*Presenter
} 


\section{Introduction}

The possible presence of various species of antimatter in the cosmic radiation can provide evidence of sources and processes important for both astrophysics and elementary particle physics. Most of the observed cosmic-ray antiprotons ( $\bar{p}$ 's) are well understood as secondary products of collisions between primary cosmic-rays and the interstellar medium. The energy spectrum of such "secondary" antiprotons peaks near $2 \mathrm{GeV}$, and decreases sharply below and above the peak, due to the kinematics of antiproton production and to the local interstellar (LIS) proton spectrum. Cosmologically "primary" sources have been suggested, including the annihilation of dark-matter particles and the evaporation of primordial black holes (PBH) by Hawking radiation [1]. While antideuterons ( $\bar{d}$ 's) have never been detected in the cosmic radiation, they can be produced by the same sources as $\bar{p}$ 's and may be of both secondary or primary origin, with the latter providing evidence for sources such as PBHs and annihilating neutralino dark matter. The low-energy range below $\sim 1 \mathrm{GeV} /$ nucleon offers a unique window in the search for cosmic-ray primary $\bar{d}$ 's because it has a greatly supressed background from secondary $\bar{d}$ 's. Thanks to their heavier mass, the probability of secondary production in cosmic-ray interactions is much smaller, especially at low energies, because of the very low production cross-section and strict kinematical requirements. Therefore, one single $\bar{d}$ event would be a direct evidence of novel primary origins. The best limit ever achieved was reported by the BESS experiment obtained during last solar minimum period [2].

\section{BESS Program}

The BESS instrument [3, 4] was developed as a high-resolution magnetic-rigidity spectrometer for cosmic-ray antiparticles and precise measurements of the absolute fluxes of various cosmic-ray

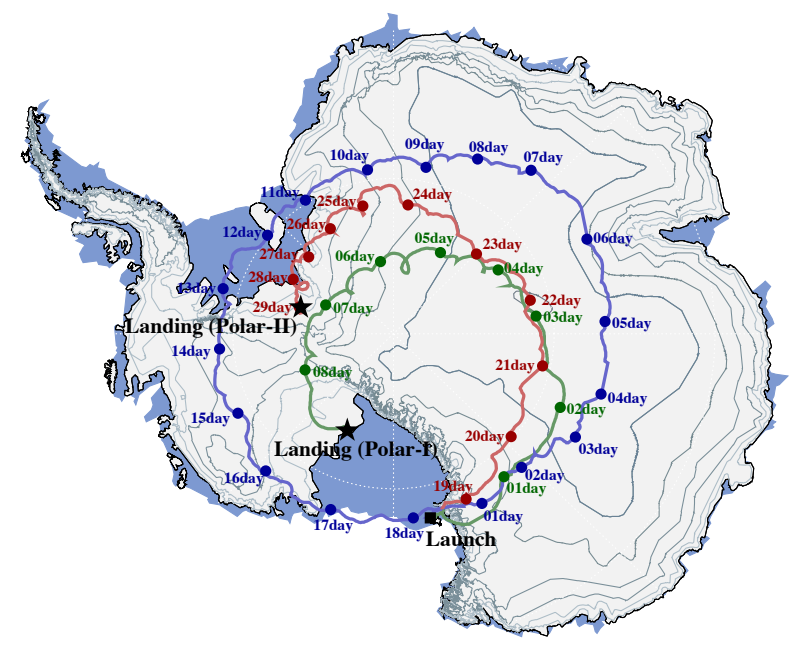

Figure 1: Flight trajectories of the 2007 BESS-Polar II over Antarctica from Williams Field (first orbit blue, second orbit red) with 2004 BESS-Polar I flight (green).

[Launch]S77-51,E166-40, 06:27(McM) 12/23 2007

[Recovery]S83-51,W073-04, 09:02(UTC) 1/21 2008 


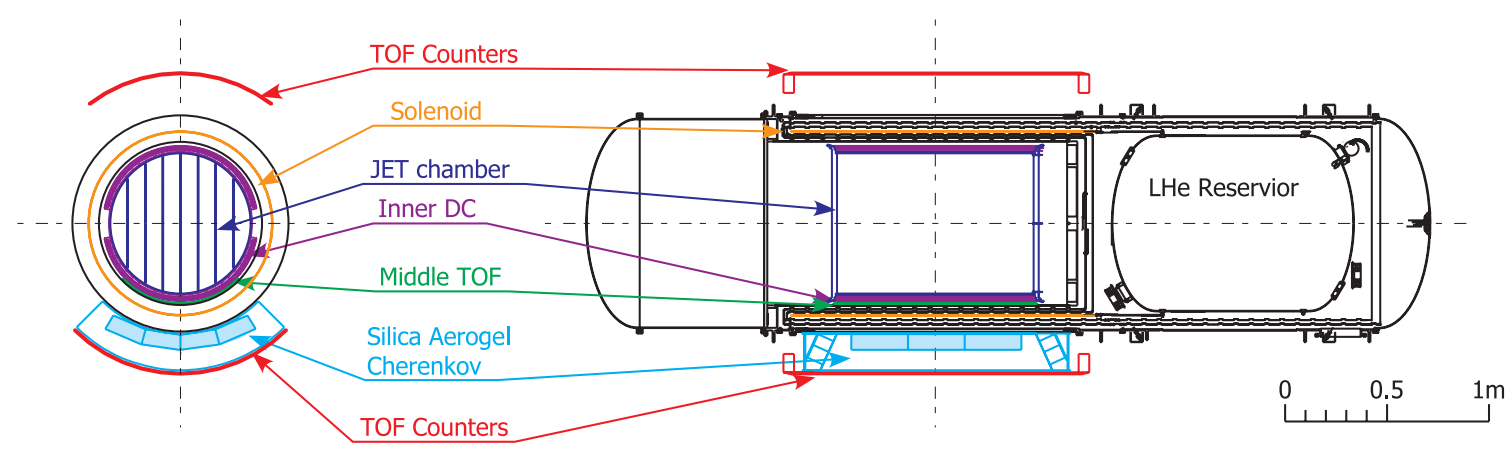

Figure 2: Cross sectional view of BESS-Polar II spectrometer

components. The original BESS experiment performed 9 flights over northern Canada during the period of 1993 through 2002 with continuous improvement in the instrument. The BESS-Polar project was proposed as an advanced BESS program using long duration balloon (LDB) flights over Antarctica (around the south pole) to provide high-statistics, low-energy cosmic-ray measurements [5-7]. The first scientific flight of the BESS-Polar instrument was launched near US McMurdo Station in Antarctica, on December 13th, 2004 (UTC). The flight duration was over 8.5 days and more than $9 \times 10^{8}$ cosmic-ray events were recorded [8]. Incorporating considerable improvements in instrument and payload systems compared to BESS-Polar I, the BESS-Polar II instrument was launched on December 23, 2007, from Williams Field near the McMurdo Station and circulated around the South Pole for 24.5 days of observation with the magnet energized. The float altitude was $34 \mathrm{~km}$ to $38 \mathrm{~km}$ (residual air of $5.8 \mathrm{~g} / \mathrm{cm}^{2}$ on average), and the cutoff rigidity was below 0.5 GV. BESS-Polar II accumulated $4.7 \times 10^{9}$ events with no inflight event selection as 13.6 terabytes of data (Fig. 1).

The BESS-Polar II program [9] has published three papers giving precise measurements of antiprotons [10], a sensitive antihelium search [11] and the absolute spectra measurements for cosmic-ray protons and helium nuclei [12]. The antiproton spectrum measured by BESS-Polar II shows good consistency with secondary antiproton calculations and no evidence of primary antiprotons originating from the evaporation of primordial black holes. The antihelium search has set a new limit in the ratio of possible antihelium to measured helium of $6.9 \times 10^{-8}$ at $95 \%$ confidence, the lowest limit to date. Proton and helium spectra observed from PAMELA [13, 14], AMS-02 $[15,16]$ and BESS-Polar agree within one $\sigma_{E}$ at high-energies.

\section{BESS-Polar Instrument}

In the BESS-Polar instruments shown in Fig. 2, a uniform magnetic field of $0.8 \mathrm{~T}$ is produced by a thin superconducting solenoid, and the field region is filled with drift-chamber tracking detectors. Tracking is performed by fitting up to 52 hit points with a characteristic resolution of $\sim 140 \mu \mathrm{m}$ in the bending plane, resulting in a magnetic-rigidity $(\equiv P c / Z e$ ) resolution of $0.4 \%$ at $1 \mathrm{GV}$ and a maximum detectable rigidity (MDR) of $240 \mathrm{GV}$. Upper and lower scintillator hodoscopes provide time-of-flight (TOF) and $d E / d x$ measurements and the event trigger. For antiproton measurements, the acceptance of BESS-Polar is $0.23 \mathrm{~m}^{2} \mathrm{sr}$ and for proton and helium measurements the acceptance is reduced to be $0.18 \mathrm{~m}^{2} \mathrm{sr}$. The timing resolution of the TOF system is $120 \mathrm{ps}$, giving a $\beta^{-1}$ 

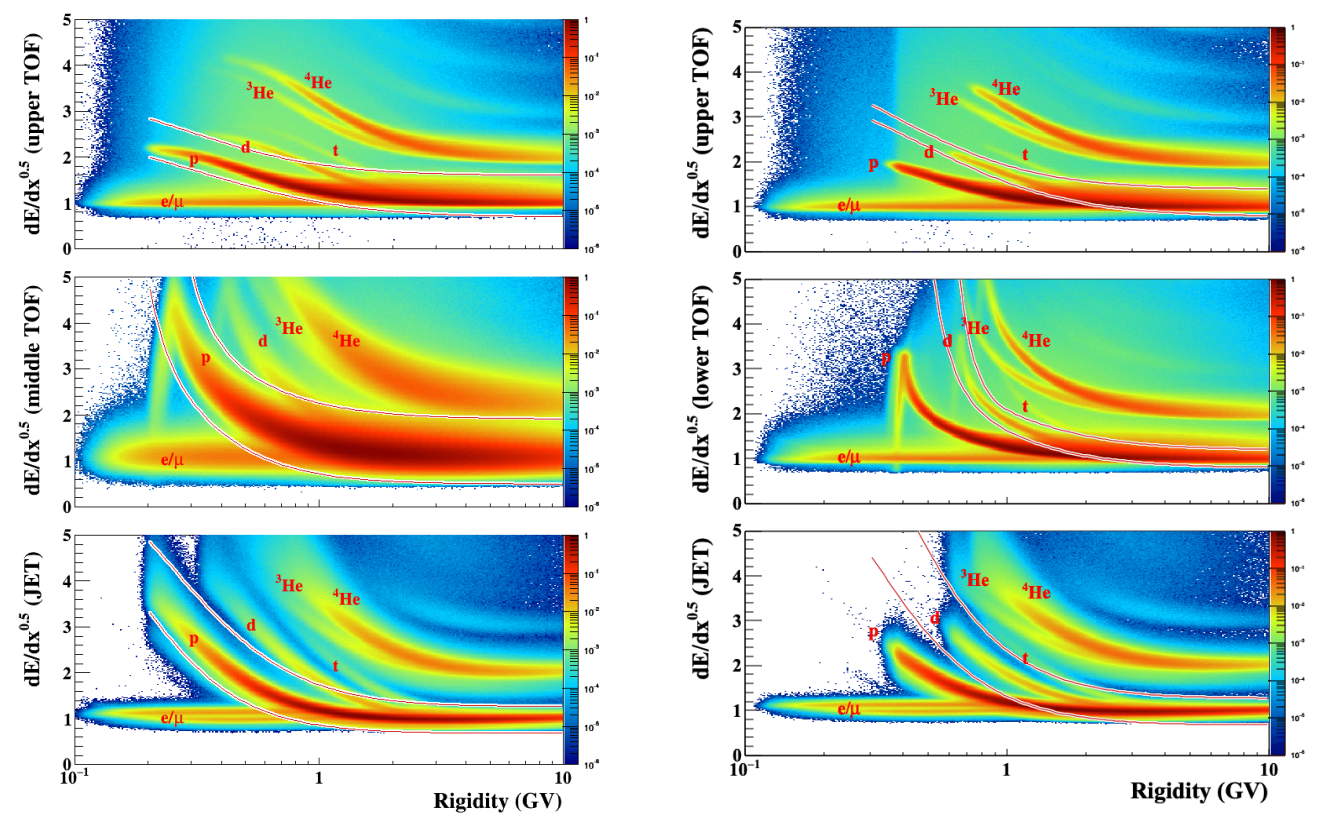

Figure 3: Proton bands in $d E / d x$ vs $R$ (top: Figure 4: Deuteron bands in $d E / d x$ vs $R$ (top: UTOF, middle: MTOF, bottom: JET) in UM UTOF, middle: LTOF, bottom: JET) in UL trigtriggered events from BESS-Polar II. gered events from BESS-Polar II.

resolution of $2.5 \%$. The instrument also incorporates a threshold-type Cherenkov counter using a silica aerogel radiator with index $n=1.03$ (ACC) that can reject $e^{-}$and $\mu^{-}$backgrounds by a factor of 12000 and distinguish $\bar{p}$ 's from such backgrounds up to $3.5 \mathrm{GeV}$. A thin scintillator middle-TOF (MTOF) is installed on the lower inner-surface of the solenoid bore enabling to detect low-energy particles which cannot penetrate the magnet wall. Since readout of both ends of the scintillators was realized for the BESS-Polar II MTOF, it has identical functionaries to the other TOF system including axial position measurements except for inferior timing resolution of $320 \mathrm{ps.}$

\section{Data analysis}

To investigate the capability for measurement of higher charge particle beyond helium in BESSPolar II, new gain stitch calibration between 18th and 13th dynode signal whose gain factor is 20 times smaller in TOF PMTs, and new method of truncated charge measurement in JET chamber were developed. Most of technique were generally for higher charge particles, but some of them such as new tracking angle correction also made the improvement of data quality for $\mathrm{Z}=1$ particles. Based on new calibrated flight data, $\bar{p}$ 's and $\bar{d}$ 's analysis were performed. As the methodology of antiparticles analysis, three different fluxes of UL triggered $\bar{p}$ 's, UL triggerred $\bar{d}$ 's and UM triggered $\vec{p}$ 's were calculated at the almost same manner to keep the consistency of cut criteria. It helps to minimize the risk of bias analysis in $\bar{d}$ 's detection study especially.

In the first stage of data analysis, we selected events with a single track fully contained inside the fiducial volume with acceptable track quality by eliminating the track which scratches the outer most region. 
A single-track event was defined as an event which has only one isolated track and one or two hit counters in each layer of the TOF hodoscopes. The single-track selection eliminated rare interacting events. To estimate the efficiency of the single-track selection, Monte Carlo simulations with GEANT4 were performed. At this point, the same selection criteria for positive and negative curvature events are applied under the assumption that non-interactive $\bar{d}(\bar{p})$ behave like $d(p)$ except for their deflection thanks to the cylindrical symmetry of the BESSPolar spectrometer. We can also estimate various efficiencies for $\bar{d}$ 's by using positive curvature events (d). The selection criteria of $\bar{d}$ are basically identical to what were applied in $\bar{p}$ analysis. To prevent $\bar{p}$ background contamination into $\bar{d}$, improved tracking quality was required by eliminating noisy IDC hit events, and selecting events that pass through center region of JET. $\bar{p}$ and $\bar{d}$ candidates are then selected
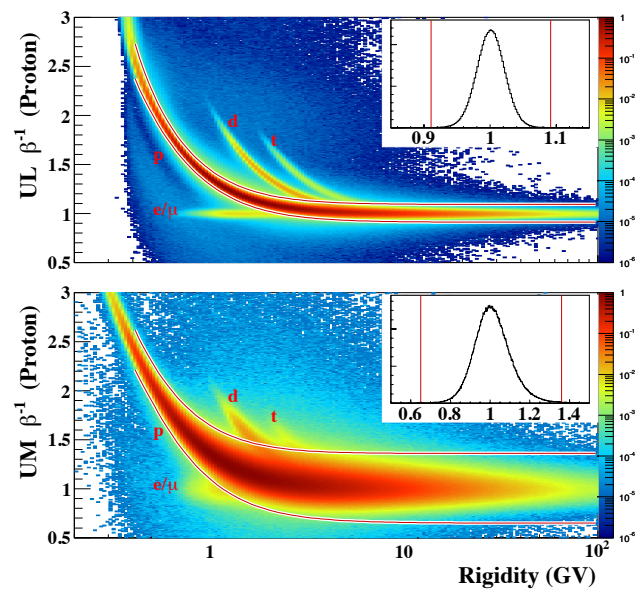

Figure 5: Scatter plot of $\beta^{-1}$ vs. rigidity obtained from the balloon observation after proton $\mathrm{dE} / \mathrm{dx}$ selection in UL (top) and UM (bottom). The superimposed graph shows the selection criteria for protons above $30 \mathrm{GV}$. by using combination of $d E / d x$ and $\beta$ information.

We first apply band selections according to $d E / d x$ for upper and middle TOF and JET chamber in UM triggered $\bar{p}$ analysis shown in Fig. 3, and lower TOF instead of middle TOF in UL triggered $\bar{d}$ analysis shown in Fig. 4. We require that $\bar{d}(\bar{p})$ as well as $d(p)$ must have the $d E / d x$ inside the band. Figure 5 shows scatter plot of $\beta^{-1}$ vs rigidity after the $d E / d x$ selections. As shown in Fig. 3, MTOF further lowers the threshold rigidity corresponding to about $100 \mathrm{MeV}$ for antiproton or proton measurements. Since the primary objective of BESS-Polar II is a precise measurement of the low-energy antiproton spectrum below $1 \mathrm{GeV}$, extending the measurement to the lowest energies is significant. Meanwhile MTOF causes large contamination of background due to inferior timing resolution of $320 \mathrm{ps}$ as compared to LTOF of 120ps ( Fig. 5 ). So $\bar{d}$ search wasn't performed in UM triggered events.

\section{Identification for UM antiproton and UL antideuteron}

\subsection{Antiproton identification}

Figure6 show $\beta^{-1}$ versus rigidity plots by incorporating data from MTOF. We see a band of about 418 antiprotons the exact mirror position of the protons. MTOF further lowers the threshold rigidity corresponding to about $100 \mathrm{MeV}$ for antiproton or proton measurements. Meanwhile MTOF causes large contamination of background due to inferior timing resolution of $320 \mathrm{ps}$ as compared to LTOF of 120ps. To prevent the background contamination of electron/muon, the antiproton candidates below the $\beta^{-1}$ threshold of 1.3 were rejected. It can exclude about $99.7 \%$ of the background. 

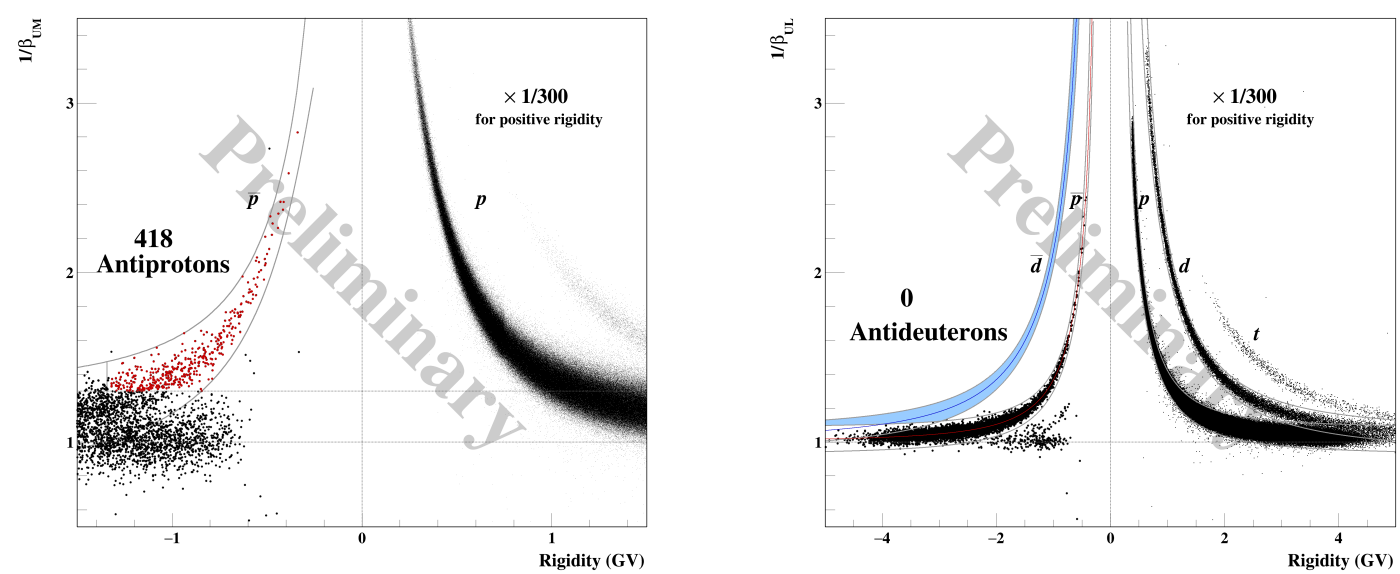

Figure 6: The $\beta_{U M}^{-1}$ versus rigidity plot and antipro- Figure 7: Surviving single-charge events in $\beta_{U L}^{-1}$ vs ton's selection band. The solid curves define the rigidity plot after $d E / d x$ and ACC cut in BESS-Polar antiproton mass bands.

II data

\subsection{Antideuteron identification}

Figure 7 shows surviving single-charge events in $\beta^{-1}$ vs rigidity plot after $d E / d x$ and ACC cut. Blue shaded band denotes signal region for $\bar{d}$ excluding $3.5 \sigma$ region from $\bar{p}$ center to prevent $\bar{p}$ contamination. In the band, we found no $\bar{d}$ candidates.

\section{Flux calculation}

\subsection{Very-low energy antiproton spectrum}

After $\bar{p}$ candidates are identified, the differential flux of $\bar{p}$ 's at the top of atmosphere $\left(\Phi_{\mathrm{TOA}}\right)$ integrated in an energy width of $\mathrm{d} E$ can be expressed as follows:

$$
\begin{array}{r}
\Phi_{\mathrm{TOA}} \mathrm{d} E=\left(N_{\mathrm{TOI}}-N_{\text {atmos }}\right) / \varepsilon_{\text {air }} /\left(S \Omega \cdot T_{\text {live }}\right) \\
N_{\mathrm{TOI}}=\left(N_{\overline{\mathrm{p}}}-N_{\mathrm{BG}}\right) /\left(\varepsilon_{\mathrm{det}} \cdot \varepsilon_{\text {non-int }}\right)
\end{array}
$$

where $T_{\text {live }}$ is the live time, $N_{\overline{\mathrm{p}}}$ and $N_{\mathrm{BG}}$ are the numbers of observed $\bar{p}$ candidates and expected background particles among the candidates. The effective geometrical acceptance including noninteraction efficiency $\left(S \Omega \cdot \varepsilon_{\text {non-int }}\right)$ was calculated using GEANT4. The detection efficiency of $\bar{p}$ 's $\left(\varepsilon_{\mathrm{det}}\right)$ was calculated using a noninteracting proton sample. Estimates of atmospheric secondaries included in $N_{\text {TOI }}\left(N_{\text {atmos }}\right)$, and a correction for survival probability in the residual atmosphere $\left(\varepsilon_{\text {air }}\right)$ were calculated by solving the transfer equations. To demonstrate the uniformity of normalization in the flux calcution, Fig. 8 shows the detection efficiency at the top panel, and the effective geometrical acceptance at the bottom panel. Except for the step structure of aerogel efficiency around $0.3 \mathrm{GeV}$ by excluding the cut for higher statistics, the normalization factors don't show a strong dependency on the kinetic energy.

\subsection{Upper limit on antideuteron flux}

In the almost same manner as antiproton analysis, the upper limit on the antideuteron flux was calcuclated because no antideuteron candidate was found. As the number of the observed 

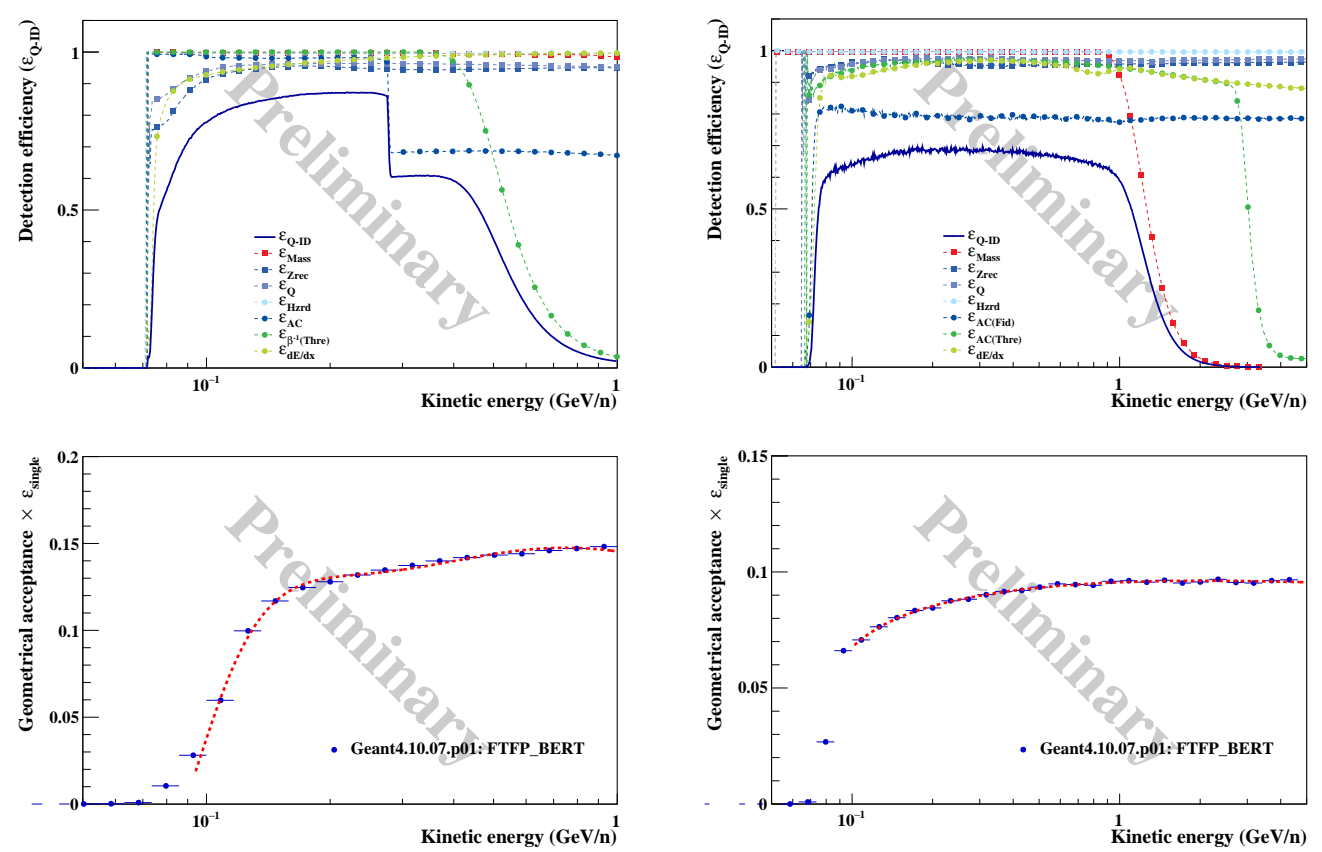

Figure 8: The detection efficiency (top) and the Figure 9: The detection efficiency (top) and the effective geometrical acceptance (bottom) in the effective geometrical acceptance (bottom) in the calculation for UM antiproton flux calculation for UL antideuteron upper limit

antideuteron events, 3.1 was taken for the calculation of the $95 \%$ C.L. upper limit. Figure 9 shows the detection efficiency at the top panel, and the effective geometrical acceptance at the bottom panel. The aerogel threshold cut was applied in whole region for antideuteron conservative analysis.

\section{Conclusion}

We will report the antideuteron search advanced in BESS-Polar II, with 95\% C.L. upper limit on the deuteron flux, and the absolute spectrum of the cosmic-ray antiproton in the lowest energy range 0.1 to $0.7 \mathrm{GeV}$ obtained in the consistent data analysis.

\section{Acknowledgements}

The BESS-Polar program is a Japan-United States collaboration, supported in Japan by the Grant-in-Aid 'KAKENHI' for Specially Promoted and Basic Researches, MEXT-JSPS, and in the U.S. by NASA. Balloon flight operations were carried out by the NASA Columbia Scientific Balloon Facility and the National Science Foundation United States Antarctic Program. We would like to express our sincere thanks for their continuous professional support.

\section{References}

[1] S. W. Hawking, Commun. Math. Phys. 43, 199 (1975). 
[2] H. Fuke et al., Phys. Rev. Lett. 95, 081101 (2005).

[3] Y. Ajima et al. (BESS), Nucl. Instrum. Meth. A443, 71 (2000).

[4] S. Haino et al., Nucl. Instrum. Meth. A518, 167 (2004).

[5] A. Yamamoto et al., Adv. Space Res. 30, 1253 (2002).

[6] J. W. Mitchell et al., Nucl. Phys. (Proc. Suppl.) 134, 31 (2004).

[7] T. Yoshida et al., Adv. Space Res. 33, 1755 (2004).

[8] K. Abe et al., Phys. Lett. B670, 103 (2008).

[9] K. Abe et al., Advances in Space Research (In Press) (2016), http://dx.doi.org/10.1016/j.asr.2016.11.004.

[10] K. Abe et al., Phys. Rev. Lett. 108, 051102 (2012).

[11] K. Abe et al., Phys. Rev. Lett. 108, 131301 (2012).

[12] K. Abe et al., Astrophys. J. 822, 65 (16pp) (2016).

[13] O. Adriani et al., Science 332, 69 (2011).

[14] O. Adriani et al., Phys. Rep. 544, 323 (2014).

[15] M. Aguilar et al., Phys. Rev. Lett. 114, 171103 (2015).

[16] M. Aguilar et al., Phys. Rev. Lett. 115, 211101 (9pp) (2015). 